The objective of the national program for poliomyelitis eradication is to keep Algeria as a zone free of Polio and to obtain the WHO certificate. In the present work, we describe the epidemiological and clinical characteristics of AFP: age, gender, autonomic disorders, albuminocytologic dissociation of CSF, artificial ventilation, and mortality.

Materials and methods Prospective analysis of all cases of AFP from September 1994 to June 2013. Since 1997, we began systematically sending: Written statements of AFP on special forms to the Algerian Health Ministry and stools culture for the Polio virus to Algerian's Pasteur Institute.

AFP was diagnosed clinically through historical and clinical examination. The underlying cause of AFP was investigated by appropriate laboratory tests, such as serum electrolytes, cerebrospinal fluid analysis, nervous conduction study and stools culture for Polio virus.

Results We report 198 cases of AFP. No case of poliomyelitis has been diagnosed. Mechanical ventilation was necessary in more than $30 \%$ of cases. Since 2008, use of intravenous immunoglobulin (IV IG) seems to improve the mortality of AFP. $8 \%$ $(n=198)$ vs. $4 \%(n=47)$. All deaths are related to the existence of severe neuro-vegetative disorders.

The last case of poliomyelitis reported, in Algeria, was in ILLIZI (extreme south east) in 1996.

Conclusions Eradication of Poliomyelitis is a priority of public health in Algeria.

\section{0-083a COAGULASE-NEGATIVE STAPHYLOCOCCAL INFECTIONS IN UK NEONATAL UNITS (NNUS)}

${ }^{1} \mathrm{~A}$ Kent, ${ }^{1} \mathrm{C}$ Kortsalioudaki, ${ }^{2} \mathrm{~T}$ Watts, ${ }^{3} \mathrm{P}$ Satodia, ${ }^{4} \mathrm{~N}$ Kennea, ${ }^{5} \mathrm{~N}$ Embleton, ${ }^{6} \mathrm{P}$ Clarke, ${ }^{7} \mathrm{~J}$ Chang, ${ }^{8} \mathrm{R}$ Geethanath, ${ }^{9} \mathrm{~T}$ Scorrer, ${ }^{1} \mathrm{PT}$ Heath. ${ }^{1}$ Paediatric Infectious Diseases Research Group Clinical Sciences, St George's University of London, London, UK; ${ }^{2}$ Neonatal Services, Evelina London Children's Hospital Guy's and St Thomas' NHS Foundation Trust, London, UK; ${ }^{3}$ Neonatal Services, University Hospital of Coventry and Warwickshire, Coventry, UK; ${ }^{4}$ Neonatal Services, St George's Healthcare Trust, London, UK; ${ }^{5}$ Newcastle Neonatal Services, Royal Victoria Infirmary Newcastle Upon Tyne, Newcastle, UK; ${ }^{6}$ Neonatal Services, Norfolk and Norwich University Hospital, Norwich, UK; ${ }^{7}$ Neonatal Services, Croydon University Hospital, London, UK; ${ }^{8}$ Neonatal Services, City Hospitals Sunderland (Sunderland Royal Hospital), Sunderland Tyne and Wear, UK; ${ }^{9}$ Neonatal Services, Queen Alexandra Hospital, Portsmouth, UK

10.1136/archdischild-2014-307384.151

Background and aims Advances in neonatal intensive care have resulted in improved survival and outcomes of increasingly preterm and very-low-birth-weight (VLBW) neonates. Invasive interventions contribute to higher rates of infections in these infants.

Abstract 0-083a Table 1 Median (IQR). LOS: late-onset sepsis, occurring after 48-hours of age. PNA: postnatal age. CRP: maximum CRP within $48 \mathrm{~h}$ of the positive culture. CVC: central-venous catheter. CVC removal: removal of the CVC due to the infection episode

\begin{tabular}{|l|c|}
\hline & Infection Episodes (N=440) \\
\hline Sex (male), $\mathrm{n}$ & $272(62 \%)$ \\
\hline Gestation (weeks) & $26(25-30)$ \\
\hline Birth weight (g) & $858(670-1285)$ \\
\hline PNA (days) & $14(8-31)$ \\
\hline LOS, $\mathrm{n}$ & $423(96 \%)$ \\
\hline C-reactive protein (mg/L) & $53(25-90)$ \\
\hline CVC in-situ, $\mathrm{n}$ & $400(91 \%)$ \\
\hline CVC removal, $\mathrm{n}$ & $210(48 \%)$ \\
\hline IUGR, $\mathrm{n}$ & $98(22 \%)$ \\
\hline
\end{tabular}

This study aims to describe infections with coagulase-negative Staphylococci (CoNS) in UK NNUs participating in a neonatal infection surveillance network (neonIN).

Methods neonIN is a web-based surveillance system (www. neonin.org.uk). Details of CoNS cases between January 2010 and May 2014 were extracted. Episodes were considered clinically significant if the infant was treated with at least 5 days of appropriate antibiotics and CRP was $>10 \mathrm{mg} / \mathrm{L}$. Repeated growth within 10 days was considered the same episode.

Results 440 episodes met the study inclusion criteria (399 infants, 23 NNUs). Incidence was 7.7/1000 NNU-admissions. Baseline characteristics for these episodes are shown in table-1. Resistance data were available for 250/440 (56.8\%) episodes with high rates of resistance to flucloxacillin $89.9 \%(169 / 188)$ and penicillin $94.4 \%(67 / 71)$ and moderate resistance to teicoplanin $20.3 \%(14 / 69)$. Susceptibility to vancomycin was high 98.3\% (169/172).

Conclusions To our knowledge, this is the largest reported cohort of neonatal CoNS infections. Most babies were $<34$ weeks gestation and $<2000 \mathrm{~g}$ birth-weight and had a CVC insitu which was removed in around half of episodes. Prevention, as well as improved strategies for identifying significant CoNS infections should be prioritised.

On behalf of the Neonaotal Infectiion Surveillance Network (neonlN).

\section{Nutrition}

\section{0-084 URSODEOXYCHOLIC ACID FOR PARENTERAL NUTRITION ASSOCIATED LIVER DISEASE IN NEONATES: A RANDOMISED CONTROLLED PILOT STUDY}

II Mohamed, ${ }^{2} \mathrm{M}$ Thibault, ${ }^{2} \mathrm{~J}$ Malo, ${ }^{2} \mathrm{E}$ Ferreira. ${ }^{1}$ Pediatrics - Neonatology, University of Montreal, Montreal, Canada; ${ }^{2}$ Pharmacy - Neonatology, University of Montreal, Montreal, Canada

\subsection{6/archdischild-2014-307384.152}

Background In neonates, parenteral nutrition associated liver disease (PNALD) is a well-documented complication. Few retrospective studies suggested that ursodeoxycholic acid (UDCA) may shorten cholestasis duration.

Objectives To evaluate the effect of UDCA treatment on the duration and severity of PNALD.

Design/methods A double blinded randomised controlled pilot study in a level III NICU. PNALD was defined as at least 2 values of conjugated bilirubin $\geq 34 \mu \mathrm{mol} / \mathrm{L}$. UDCA or placebo was started after randomization within $72 \mathrm{~h}$ of the diagnosis. Exclusion criteria included biliary tract anomalies, TORCH infection, short bowel syndrome, congenital hypothyroid, urinary tract infection, and congenital metabolic or genetic disorders. Baseline characteristics, clinical and biochemical evolution information

\begin{tabular}{llll} 
Abstract 0-084 Table 1 & & & \\
\hline Gestational age, mean (SD) in weeks & $274 / 7(3 / 7)$ & $276 / 7(3 / 7)$ & 0.84 \\
Birth weight, mean (SD) in grams & $1032(608)$ & $1237(696)$ & 0.43 \\
Sex (female) & $6(43 \%)$ & $5(42 \%)$ & 0.95 \\
Intrauterine groth restriction & $5(36 \%)$ & $1(8 \%)$ & 0.12 \\
Respiratory distress syndrome & $12(86 \%)$ & $10(83 \%)$ & 0.64 \\
Apnea on Caffeine & $10(71 \%)$ & $10(83 \%)$ & 0.4 \\
Surgical intervention before PNALD & $4(29)$ & $4(33 \%)$ & 0.56 \\
\hline
\end{tabular}

